\title{
LES COPÉPODES PARASITES DE MOLLUSQUES
}

\author{
Par Théodore MONOD et Robert-Ph. DOLLFUS
}

\section{Premier supplément}

Dans le dernier numéro de ces Annales ( $\mathrm{n}^{\circ} 2$, mars 1932, p. 204), nous avons mentionné deux exemplaires d'un Copépode parasite inédit et en avons annoncé la description.
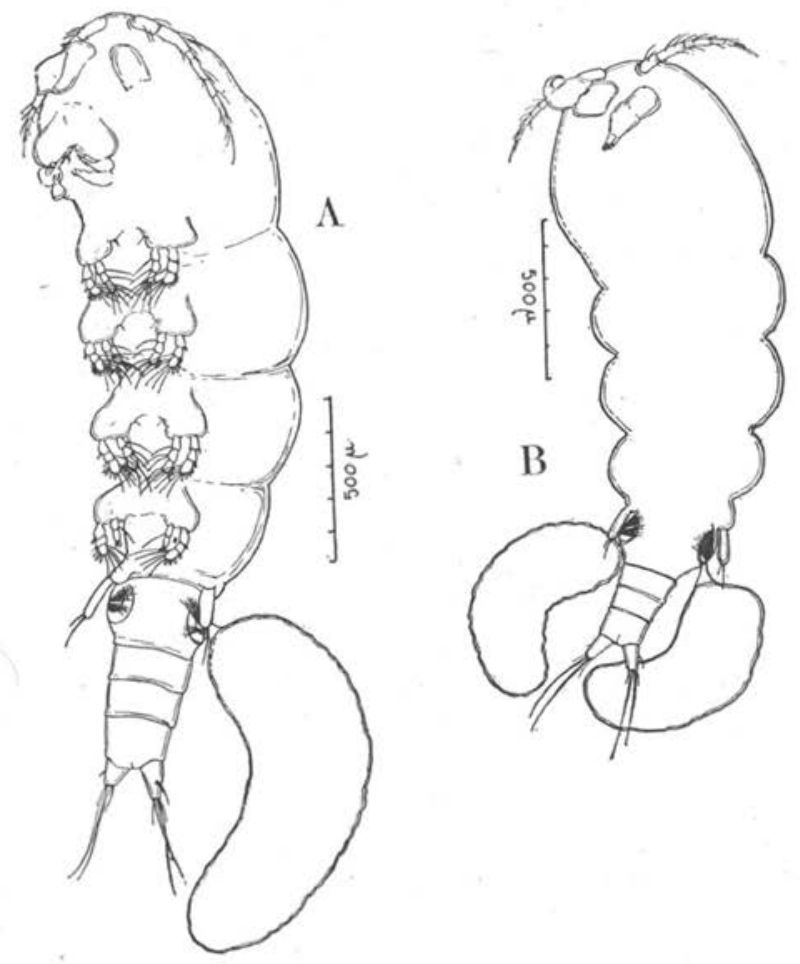

F1G. 1. - Strongylopleura pruvoli n. sp. - A-B. Les deux spécimens, $\alpha$ et $\beta$.

Strongylopleura (?) pruvoti n. sp. nom. prov.

Localité. -2 spécimens, $q$ ovigères $(2,4$ et $1,9 \mathrm{~mm}$.) ; localité et hòte inconnus. Les exemplaires, qui nous ont aimablement été remis par Mme A. Pruvot-Fol, ont probablement été recueillis dans un mollusque. Nous dédions l'espèce à Mme Pruvot.

Annales de Parasitologie, T. $\mathrm{X}, \mathrm{N}^{\circ} 3 .-1^{\mathrm{er}}$ mai 1932 , p. 295-299. 
Description. - Corps allongé, étroit, légèrement claviforme, étant renflé à son extrémité céphalique. Céphalothorax indistinctement séparé du thorax, largement arrondi en avant. Somites thoraciques libres indistinctement séparés mais bien marqués latéralement par une succession de dilatations arrondies du contour somatique ; dernier somite thoracique très réduit. Abdomen 4-arti-

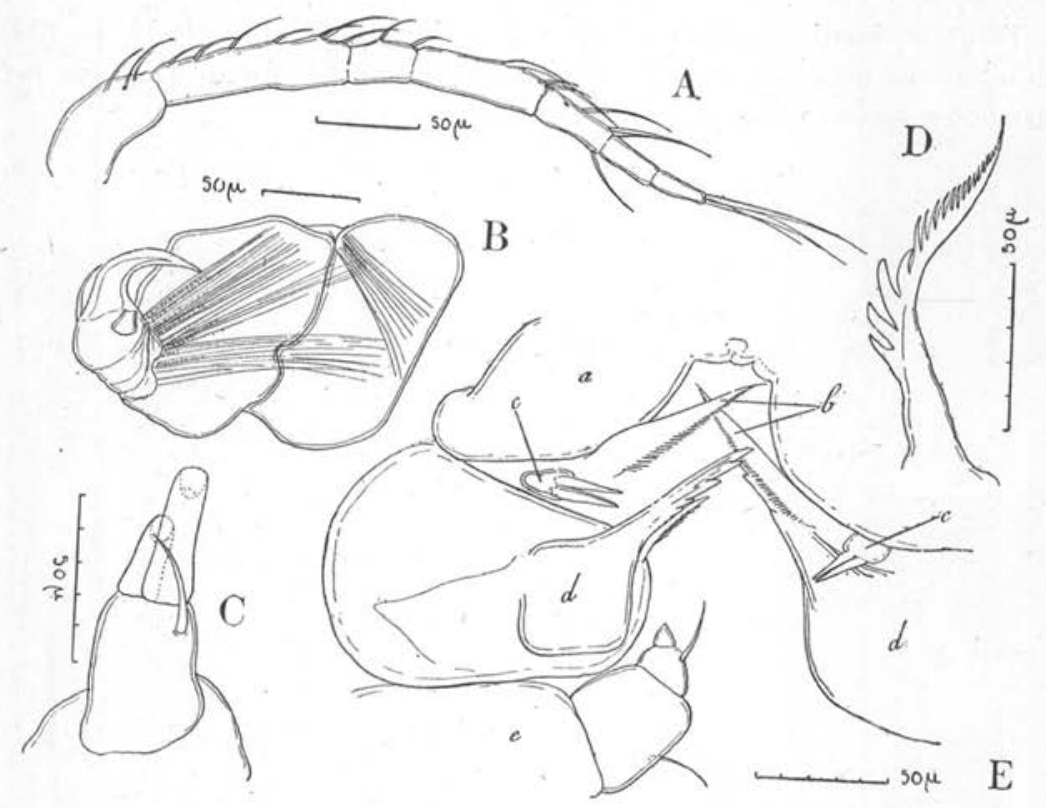

FIG. 2. - A. Antennule ( $\beta)$. - B. Antenne $(\beta)$ - - C. Extrémité de l'autre antenne $(\beta)$. - D, Partie distale de la maxille gauche $(\alpha)$. - E. Pièces buccales $(\beta): a$. labrum, $b$. mandibules, $c$. maxillules, $d$. maxilles, $e$, maxillipède droit.

culé, très court, n'atteignant pas même en longueur la moitié de celle de la partie antérieure du corps ; somite génital légèrement dilaté, sensiblement égal à la somme des deux somites suivants ; somite anal plus long que le précédent. Branches furcales sensiblement égales au somite anal; cylindro-coniques, portant une sétule marginale externe vers le milieu de leur longueur et, apicalement, 2 soies et 2 sétules. Yeux paraissant entièrement absents. Antennule 7-articulée, à articulations assez peu distinctes, article II le plus long. Antenne pauci-articulée (3-articulées ?) extrêmement courte et robuste, portant, à son extrémité, 2 forts crochets ( 1 prin- 


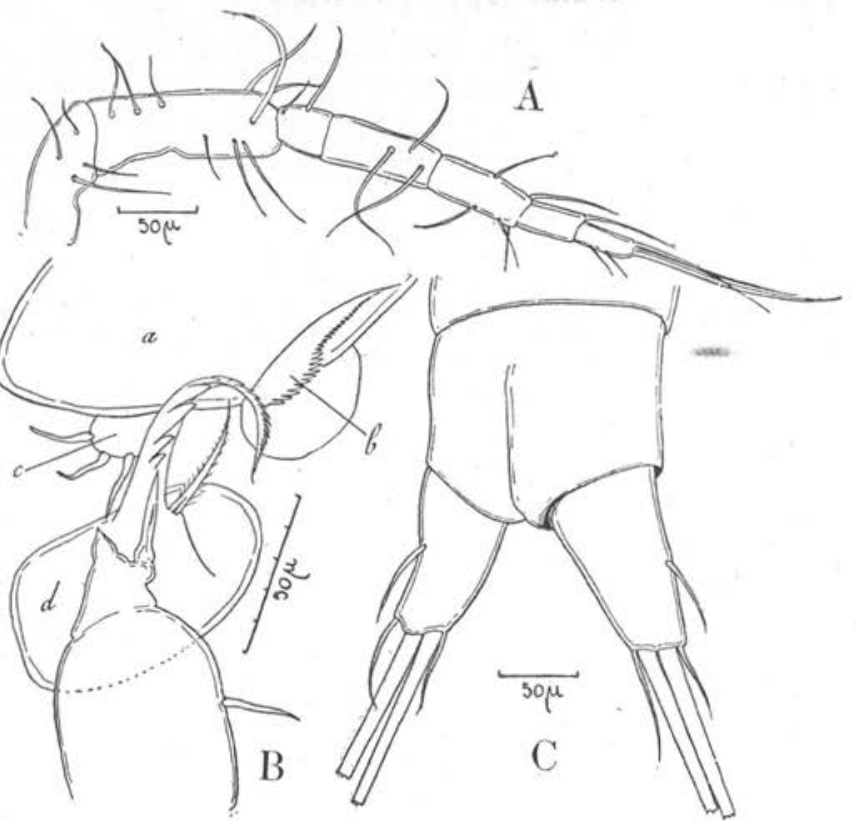

Fig. 3. - A. Antennule (a), - B. Pièces buccales (q), còté droit : a. labrum, $b$. mandibule, $c$. maxillule, $d$. maxille, e. maxillipède. - C. Extrémité caudale $(\beta)$.
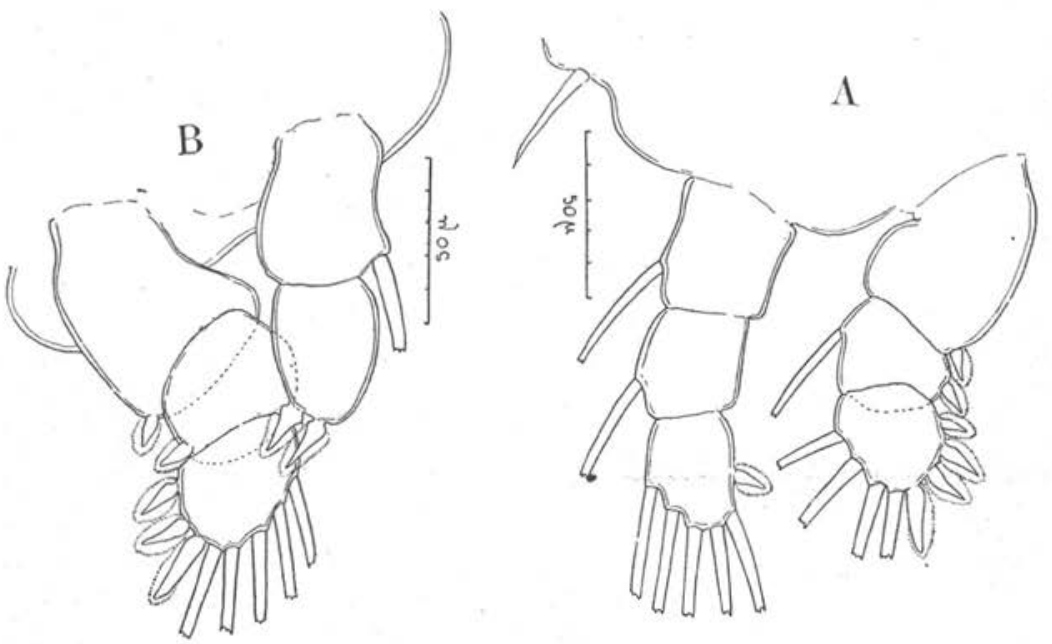

Fig. 4. - A, Patte thoracique I $(\alpha)$. - B. Patte thoracique IV $(\alpha)$. 
cipal, 1 accessoire, plus petit) et 1 sétule externe. Lèvre antérieure en forme de cloche, échancrée au milieu du bord distal dont les angles latéraux sont arrondis. Mandibules falciformes, finement
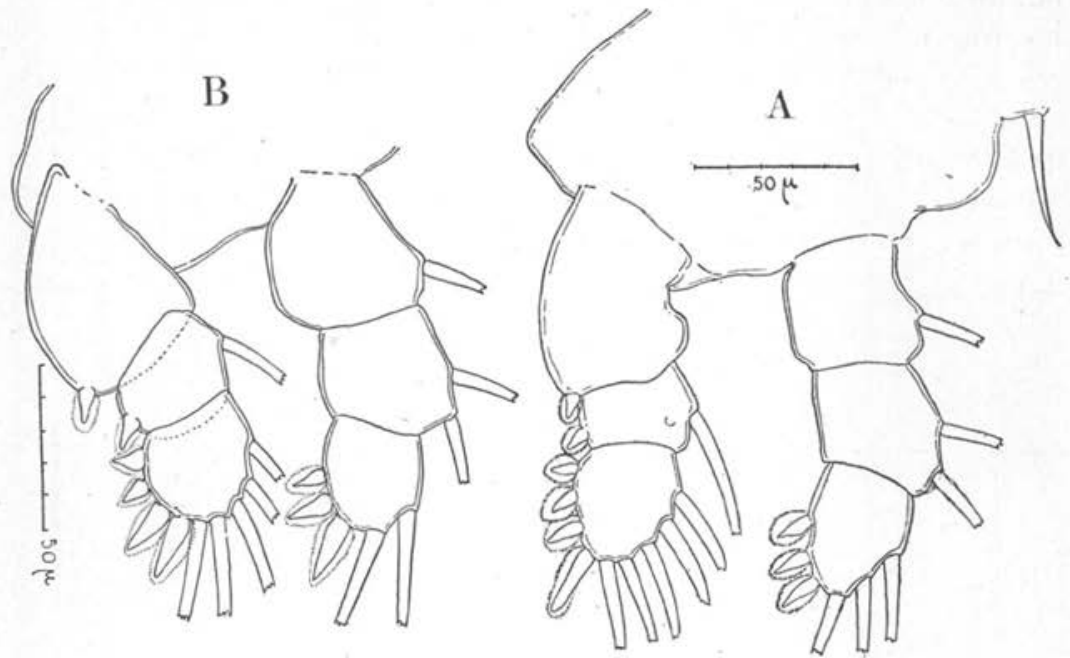

Fig. 5. - A. Patte thoracique II $(\alpha)$, - B. Patte thoracique III $(\alpha)$.
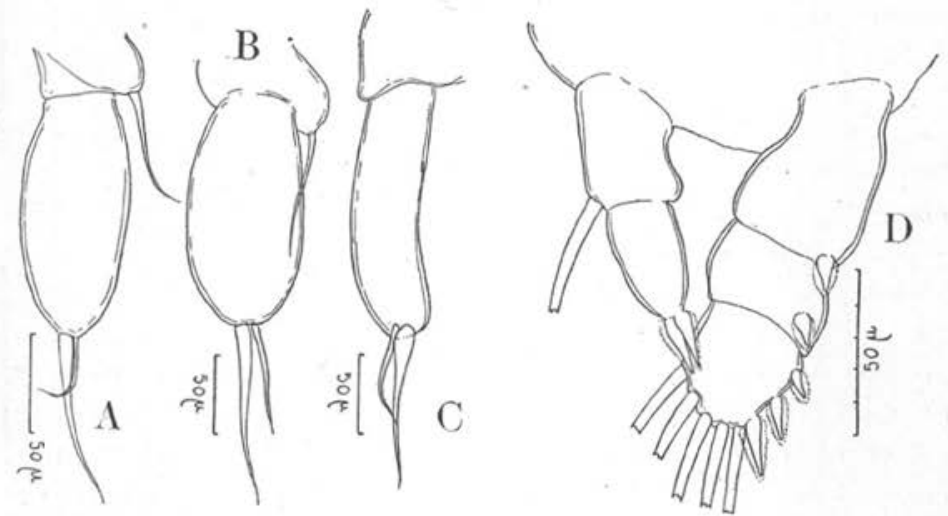

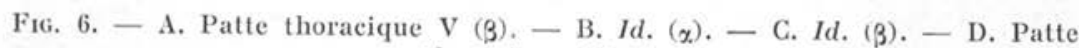
thoracique IV $(\beta)$.

pectinées, terminés en fouet. Maxillules réduites à un très petit tubercule sétigère (2-3 (?) sétules courtes) situé au niveau du bord distal de la lèvre supérieure. Maxilles relativement très développées, comprenant une partie basilaire considérable (muscles) por- 
tant une lame principale fortement denticulée et passant distalement à un fouet finement pectiné et une tige plumeuse accessoire. Maxillipèdes 4-articulés (?), article apical conique, très petit, pénultième portant 1 sétule antéro-interne, antépénultième avec 1 sétule marginale interne. Pattes thoraciques au nombre de 4 paires ; exopodites 3-articulés partout, endopodites 3- (I-III) ou 2-articulés (IV) ; V 1-articulée, ovale-allongée, avec 2 soies apicales, inégales; les épines des pattes thoraciques sont courtes et entourées d'une très large frange striée. Chétotaxie (épines en chiffres romains, soies en chiffres arabes):

\begin{tabular}{|c|c|c|c|c|c|c|c|c|c|c|c|c|c|c|c|}
\hline \multicolumn{8}{|c|}{ BORD EXTERNE } & \multicolumn{8}{|c|}{ BoHd INTERNE } \\
\hline \multicolumn{4}{|c|}{ Exopodite } & \multicolumn{4}{|c|}{ Endopodite } & \multicolumn{4}{|c|}{ Exopodite } & \multicolumn{4}{|c|}{ Endopodite } \\
\hline I & I & I & I & $\mathrm{O}$ & $\mathrm{O}$ & $\mathrm{O}$ & $\mathrm{O}$ & 0 & 0 & 0 & 0 & 1 & 1 & 1 & 1 \\
\hline I & 1 & I & I & $\mathrm{O}$ & $\mathrm{O}$ & $\mathrm{O}$ & II (1) & 1 & 1 & 1 & 1 & 1 & 2 & 2 & II (1) \\
\hline IV & IV & IV & III & I & III & III & & 4 & 5 & 5 & 5 & 5 & 3 & 2 & \\
\hline & & & & & & $A T$ & ES TH & RA & $\mathrm{IQU}$ & & & & & & \\
\hline 1 & 2 & 3 & 4 & 1 & 2 & 3 & 4 & 1 & 2 & 3 & 4 & 1 & 2 & 3 & 4 \\
\hline
\end{tabular}

Sacs ovigères latéraux, botuliformes, légèrement arqués, dépassant en arrière l'extrémité caudale.

Remarques. - Il est, pour l'instant, tout à fait impossible de déterminer avec précision la position systématique de cette espèce. Elle semble se rapprocher - au moins extérieurement - des genres Clausia Claparède et Myicola Wright, mais la présence d'un maxillipède développé chez la femelle paraît devoir l'écarter cependant de la famille des Clausiidær. Il s'agirait donc probablement d'un Lichomolgidé et, bien que les descriptions de Pelseneer ne fassent aucune allusion aux pièces buccales, on remarquera des ressemblances certaines entre l'aspect de notre espèce et celui des genres Leptinogaster et Strongylopleura de Pelseneer; en fait, c'est à ce dernier genre, qu'avec doute et seulement à titre provisoire, nous rattachons l'espèce recueillie par Mme A. Pruvot-Fol.

Laboraloire des pêches coloniales du Muséum Nalional d'Histoire Nalurelle de Paris.

(1) Apicales. 